\title{
Collective And Single-particle Structures In The Neutron-rich Doubly Mid-shell Nucleus ${ }^{170}$ Dy
}

\author{
P.-A. Söderström ${ }^{* a}$, P. M. Walker ${ }^{b}$, J. Wu ${ }^{a c}$, H. L. Liu ${ }^{d}$, P. H. Regan ${ }^{b e}$, H. Watanabe ${ }^{f}$, \\ P. Doornenbal ${ }^{a}$, Z. Korkulu ${ }^{g}$, P. Lee ${ }^{h}$, J. J. Liu ${ }^{i}$, G. Lorusso $^{a e}$, S. Nishimura ${ }^{a}$, \\ V. H. Phong ${ }^{a j}$, T. Sumikama ${ }^{k}$, F. R. Xu ${ }^{c}$, A. Yagi ${ }^{l}$, G. X. Zhang ${ }^{f}$, D. S. Ahn ${ }^{a}$, \\ T. Alharbi ${ }^{m}$, H. Baba ${ }^{a}$, F. Browne ${ }^{n}$, A. M. Bruce ${ }^{n}$, R. J. Carroll ${ }^{b}$, K. Y. Chae ${ }^{o}$, \\ Zs. Dombradi ${ }^{g}$, A. Estrade ${ }^{p}$, N. Fukuda ${ }^{a}$, C. Griffin ${ }^{p}$, E. Ideguchi ${ }^{l q}$, N. Inabe ${ }^{a}$, \\ T. Isobe ${ }^{a}$, H. Kanaoka ${ }^{l}$, S. Kanaya ${ }^{l}$, I. Kojouharov ${ }^{r}$, F. G. Kondev ${ }^{s}$, T. Kubo ${ }^{a}$, \\ S. Kubono ${ }^{a}$, N. Kurz ${ }^{r}$, I. Kuti ${ }^{g}$, S. Lalkovski ${ }^{b}$, G. J. Lane ${ }^{t}$, E. J. Lee ${ }^{o}$, C. S. Lee ${ }^{h}$, \\ G. Lotay ${ }^{b}$, C.-B. Moon ${ }^{u}$, I. Nishizuka ${ }^{k}$, C. R. Niţă ${ }^{n v}$, A. Odahara ${ }^{l}$, Z. Patel ${ }^{b}$, \\ Zs. Podolyák ${ }^{b}$, O. J. Roberts ${ }^{w}$, H. Sakurai ${ }^{a x}$, H. Schaffner ${ }^{r}$, C. M. Shand ${ }^{b}$, H. Suzuki ${ }^{a}$, \\ H. Takeda ${ }^{a}$, S. Terashima ${ }^{f}$, Zs. Vajta ${ }^{g}$, J. J. Valiente-Dòbon ${ }^{y}$ and Z. Y. Xu ${ }^{i}$ \\ ${ }^{a}$ RIKEN Nishina Center 2-1 Hirosawa, Wako-shi, Saitama 351-0198, Japan \\ ${ }^{b}$ University of Surrey Guildford, GU2 7XH, UK \\ ${ }^{c}$ Peking University Beijing 100871, China \\ ${ }^{d}$ Xi' an Jiaotong University, Xi'an 710049, China \\ ${ }^{e}$ National Physical Laboratory, Teddington, Middlesex, TW11 OLW, UK \\ ${ }^{f}$ Beihang University, Beijing 100191, China \\ ${ }^{g}$ Institute for Nuclear Research, P. O. Box 51, Debrecen, H-4001, Hungary \\ ${ }^{h}$ Chung-Ang University, Seoul 156-756, Republic of Korea \\ ${ }^{i}$ The University of Hong Kong, Pokfulam Road, Hong Kong \\ ${ }^{j}$ VNU Hanoi University of Science, 334 Nguyen Trai, Thanh Xuan, Hanoi, Vietnam \\ ${ }^{k}$ Tohoku University, Aoba, Sendai, Miyagi 980-8578, Japan \\ 'Osaka University, Machikaneyama-machi 1-1, Osaka 560-0043 Toyonaka, Japan \\ ${ }^{m}$ College of Science in Zulfi, Almajmaah University, P.O. Box 1712, 11932, Saudi Arabia \\ ${ }^{n}$ University of Brighton, Brighton BN2 4GJ, United Kingdom \\ ${ }^{o}$ Sungkyunkwan University, Suwon 440-746, Republic of Korea \\ ${ }^{p}$ University of Edinburgh, Edinburgh EH9 3JZ, United Kingdom \\ ${ }^{q}$ RCNP, Osaka University, Ibaraki, Osaka 567-0047, Japan \\ ${ }^{r}$ GSI Helmholtzzentrum für Schwerionenforschung GmbH, 64291 Darmstadt, Germany \\ ${ }^{s}$ Argonne National Laboratory, Argonne, Illinois 60439, USA \\ ${ }^{t}$ Australian National University, Canberra, A.C.T. 0200, Australia \\ ${ }^{u}$ Hoseo University, Asan, Chungnam 336-795, Korea \\ vIFIN-HH, RO-077125 Bucharest, Romania \\ ${ }^{w}$ University College Dublin, Belfield, Dublin 4, Ireland \\ ${ }^{x}$ University of Tokyo, Hongo, Bunkyo-ku, Tokyo 113-0033, Japan \\ ${ }^{y}$ Istituto Nazionale di Fisica Nucleare, Laboratori Nazionali di Legnaro, 35020 Legnaro, Italy \\ E-mail: pasoderaribf.riken.jp
}


One of the most successful descriptions of the structure of atomic nuclei is the spherical shell model. It, however, becomes impractical when moving away from closed-shell nuclei. Instead, it is the interplay between the macroscopic shape degrees of freedom and the microscopic nature of the underlying single-particle structure in a deformed basis that determines the nuclear structure. Being the heaviest nucleus precisely in the middle of, known, closed proton and neutron shells, ${ }^{170}$ Dy has become a central calibration point for tests of collective models of nuclear physics. However, besides one candidate transition from a previous experiment in Legnaro, Italy, no experimental information is available for this nucleus. Using the EURICA setup at RIKEN, which couples the worlds highest intensity in-flight fission facility with a high-efficiency HPGe array, an experiment in November 2014 produced ${ }^{170}$ Dy nuclei by in-flight fission of a ${ }^{238} \mathrm{U}$ beam. The results from this experiment provide a wealth of information on this elusive nucleus, including the evolution of quadrupole collectivity, rigidity and higher order deformations, as well as the long sought for isomeric $K=6^{+}$state, predicted to be exceptionally pure at mid-shell. These results provide us with a rich level scheme for discussing both single-particle and collective structures at mid-shell.

The 26th International Nuclear Physics Conference

11-16 September, 2016

Adelaide, Australia

* Speaker. 


\section{Introduction}

One of the most successful approaches to the nuclear many-body problem is the the spherical shell model. However, despite being a powerful model for certain regions of the nuclear chart, it becomes impractical when moving away from closed-shell nuclei. Instead, it is the interplay between the macroscopic shape degrees of freedom and the microscopic nature of the underlying single-particle structure of shell-model orbitals in a deformed basis that offers an explanation for the observed nuclear structure. The regions in the Segré chart where quadrupole collectivity is most prominent are around the doubly mid-shell nuclei, with many valence particles and far away from closed shells. Neglecting any potential sub-shell closures, the nucleus with $A<208$ that has the largest number of valence particles is ${ }_{66}^{170} \mathrm{Dy}_{104}$, lying precisely in the middle of the closed proton $Z=50,82$ and neutron $N=82,126$ shells, with $Z=66$ and $N=104$. Thus, ${ }^{170}$ Dy has become a central calibration point for tests of collective as well as single-particle models [1, 2, 3, 4]. The amount of collectivity has been shown to have a smooth dependence on both the energy of the first excited state, $E\left(2^{+}\right)$, and the reduced transition probability from the first state to the ground state, $B\left(\mathrm{E} 2: 2^{+} \rightarrow 0^{+}\right)$, as well as the energy ratio of the first excited $4^{+}$and $2^{+}$states, $E\left(4^{+}\right) / E\left(2^{+}\right)$. Indeed, this region in the nuclear chart covers the largest area where known data show very smooth collective behavior, as illustrated in Figure 1.
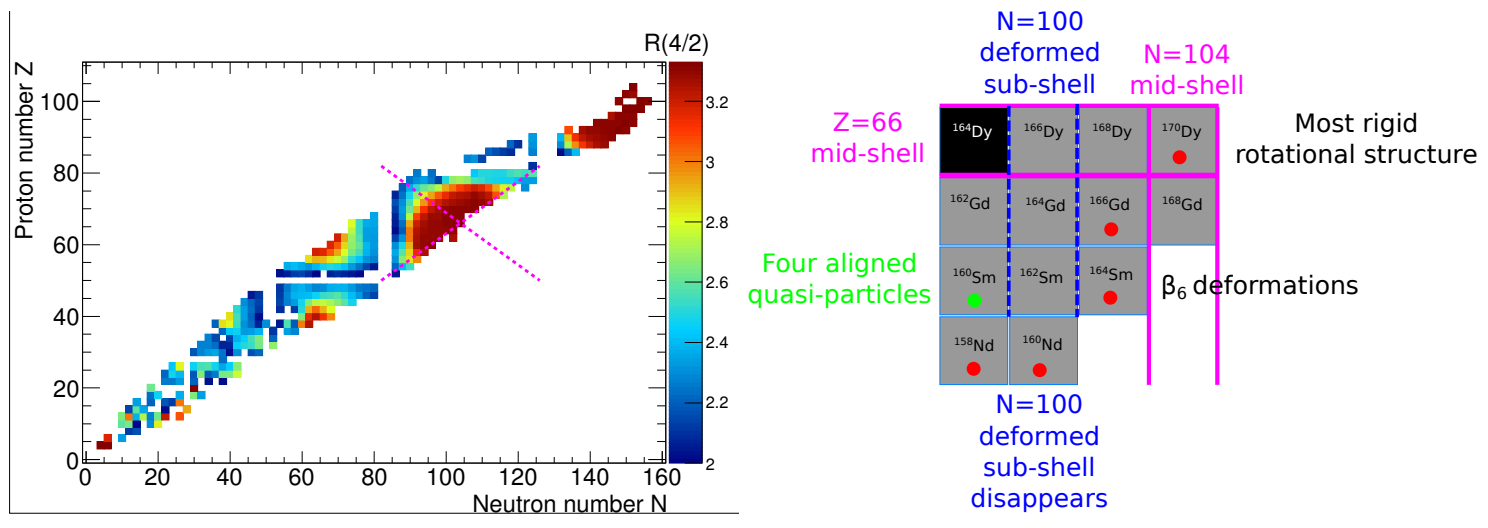

Figure 1: Systematics of the energy ratios of the first $4^{+}$and $2^{+}$excited states (left) with ${ }^{170}$ Dy marked by dashed magenta lines. In the right panel is a enlargement of the region immediately below $N=104$ and $Z=66$, together with some recent physics studies from this region highlighted.

What speaks against this simplistic picture are possible deformed and spherical sub-shell closures and other deviations from the smooth systematics that are observed in, for example, ${ }^{190} \mathrm{~W}$ $[5,6,7]$ and along the $N=100$ isotone chain $[8,9,10]$, the latter illustrated in Figure 1. Indeed, some theoretical studies predict that the quadrupole deformation maximum occurs below the $N=104$ mid-shell neutron number within an isotope chain $[4,3,11]$, while experimental data indicate that the deformation increases as $Z$ decreases below mid-shell $[9,10]$.

From a single-particle point of view, one predicted property of ${ }^{170} \mathrm{Dy}$ is the long-lived $K^{\pi}=6^{+}$ two quasi-particle isomer $[3,4,12]$, where $K$ is the total angular momentum projection on the prolate symmetry axis. The structural and decay properties of this kind of isomeric states serve as a sensitive test of the structural evolution in the well deformed nuclei. for example, recent work with EURICA shows how one can use such isomers to probe higher-order deformations [13] and how 
neutron- and proton quasi-particles together can form highly hindered high-spin isomeric states, also illustrated in Figure 1. In particular, the reduced hindrance of an isomeric state can provide information about the purity of the isomeric state and the nuclear rigidity versus the amount of mixing or $\gamma$ softness exhibited.

\section{Experiment}

During November 2014 an experiment was carried out at the RIBF at RIKEN to study ${ }^{170}$ Dy, where these nuclei as well as the $\beta$-decay parent nuclei ${ }^{170} \mathrm{~Tb}$ were produced by in-flight fission of a $345 \mathrm{MeV} / \mathrm{u}{ }^{238} \mathrm{U}$ beam with $10 \mathrm{pnA}$ intensity, incident on a Be target [14]. The experiment was carried out with two BigRIPS separator settings: 13.5 hours focusing on ${ }^{170}$ Dy ( $~ 10000$ implantations); and 45 hours focusing on ${ }^{172} \mathrm{Dy}$, during which $\sim 2500{ }^{170} \mathrm{~Tb}$ nuclei were implanted. The fragments were separated and identified in the BigRIPS separator and the ZeroDegree spectrometer [15] event-by-event, based on their mass-to-charge ratio $(A / q)$ and atomic number $(Z)$. At the final focal plane of the beam line they were implanted in the WAS3ABi active stopper $[16,17]$. The $\gamma$ rays emitted following isomer and $\beta$ decay of the implanted nuclei were detected using EURICA (Euroball-RIKEN Cluster Array) [16, 18]. The total full-energy peak detection efficiency of EURICA was, in this experiment, about $9 \%$ at a $\gamma$-ray energy of $1 \mathrm{MeV}$. Implanted ions were correlated to $\beta$-decay events when occurring within $2 \mathrm{~mm}$ of each other. Due to the high contamination of lighter fragments, a large plastic scintillator was placed behind WAS3ABi and used as a veto detector for fragments passing through the silicon detector. The level scheme obtained from this experiment is shown in Figure 2

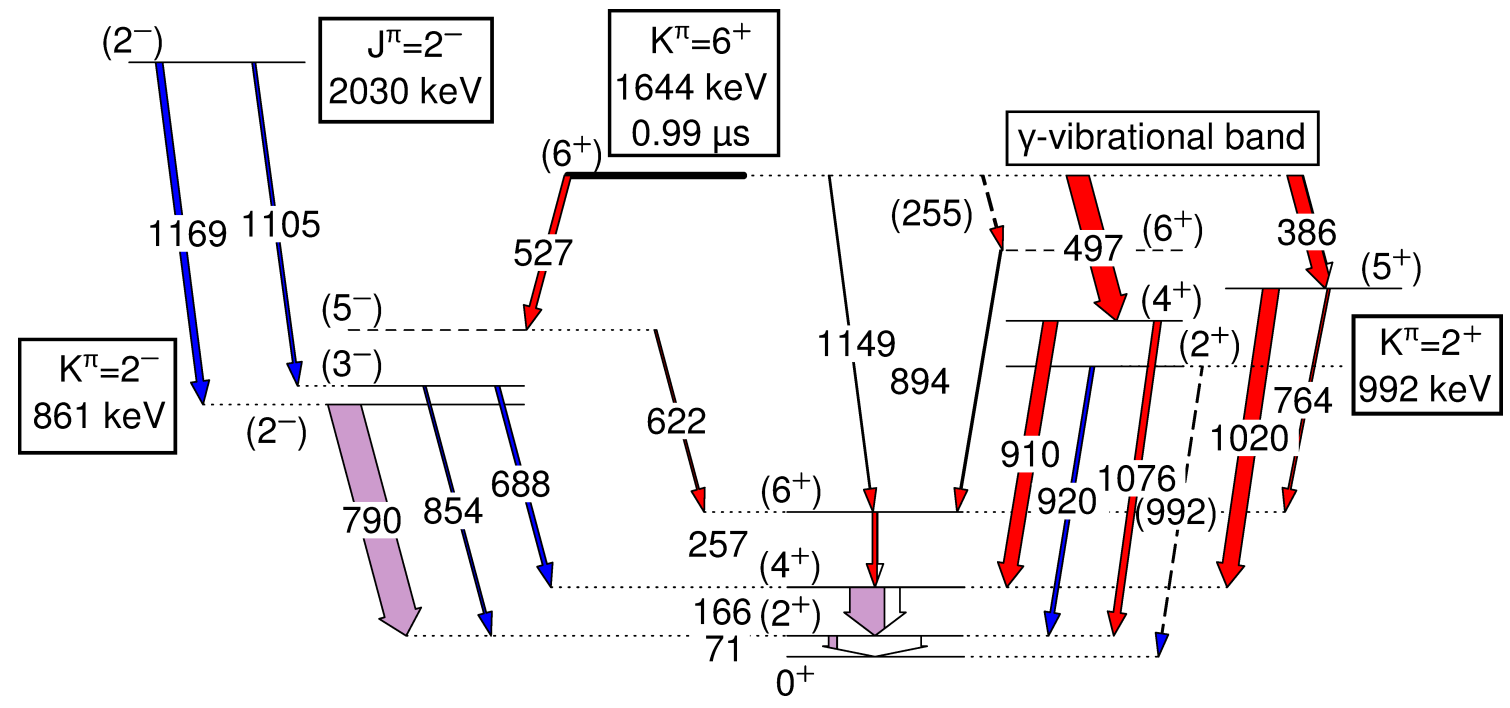

Figure 2: Partial level scheme of ${ }^{170} \mathrm{Dy}$. The thick $K^{\pi}=6^{+}$level at $1644 \mathrm{keV}$ represents the isomeric state. Unfilled parts of the arrows correspond to estimated transition strengths associated with electron conversion. This level scheme has previously been published in Reference [14].

\section{Collective Structures}

One of the aims of this experiment was the evolution of collective structures in the mid-shell 
region. Some discussion on the evolution of the yrast band has recently been published in Reference $[19,20]$. As mentioned in the introduction, the naïve picture is that the collective structures show a smooth evolution from the closed shells to mid-shell. On the other hand, self-consistent Hartree-Fock calculations with a variety of Skyrme parameterizations all suggest that the deformation maximum is expected at either ${ }^{166} \mathrm{Dy}$ or ${ }^{168} \mathrm{Dy}$ [3]. This is also consistent with the calculations made by Möller and Nix using the finite range liquid drop model [11], where ${ }^{168}$ Dy has the largest value. However, the decrease in $E\left(2^{+}\right), E\left(4^{+}\right)$, as well as the increase of $R(4 / 2)$ suggest that the collectivity and deformation continue to increase at least until $N=104$. This is more consistent with the naïve neutron mid-shell picture rather than the more comprehensive nuclear models.

Another interesting aspect is the evolution of non-yrast states as a signature of changes in collective structure. These are also expected to change smoothly with neutron number and, in particular, the second $2^{+}$are expected to highlight changes in $\gamma$ softness while the negative parity $1^{-}, 2^{-}$and $3^{-}$states are often related to octupole deformation. In Figure 3, the systematics of the known $2_{2}^{+}$and $3_{1}^{-}$in the region discussed in these proceedings are shown. It is interesting to note that these do indeed evolve rather smoothly with an on average almost constant value of $2_{2}^{+}$within the dysprosium chain above $N=90$, while the $3_{1}^{-}$states show a decreasing trend of about $50 \mathrm{keV}$ per neutron pair in the same chain.

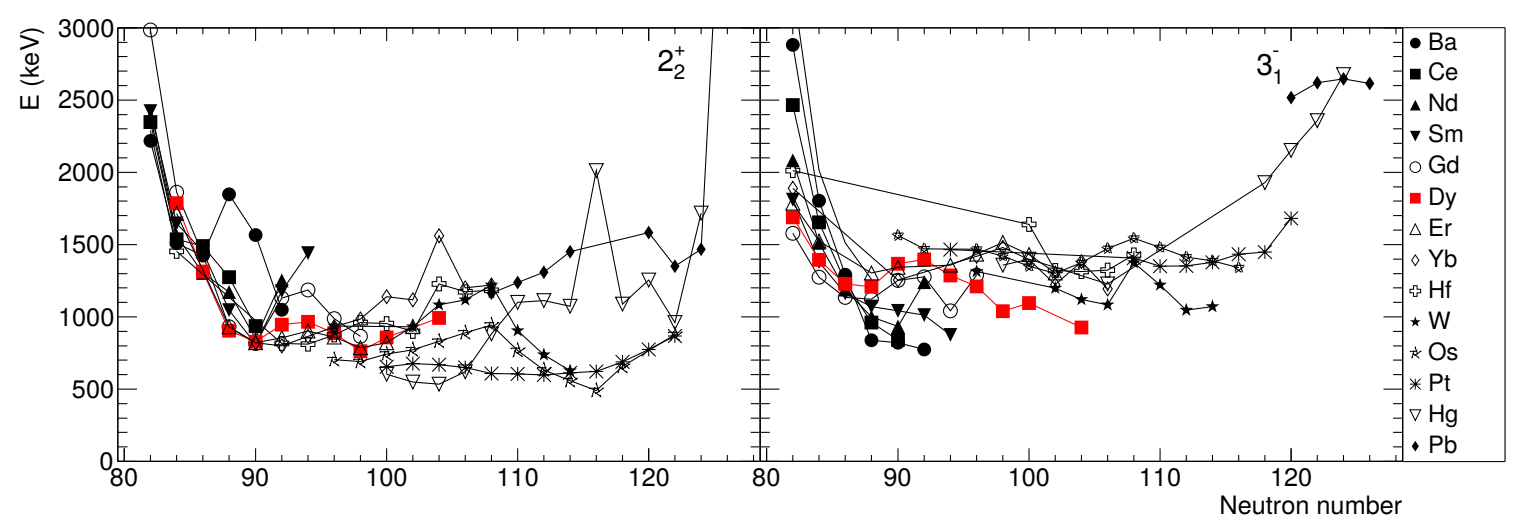

Figure 3: Systematics of the $2_{2}^{+}$(left) and $3_{1}^{-}$states (right) in the region discussed in these proceedings.

\section{Single-particle Structures}

Based on a scheme correlating collective observables to the product of valence protons and neutrons we would expect the $6^{+}$isomer to have a lifetime of $\sim 3 \mathrm{~s}$ [12], given the energy reported in Reference [14] and assuming the hindrance of all decay channels scale the same, while the measured value is just $1 \mu \mathrm{s}$, roughly six orders of magnitude shorter. While it is indeed possible to measure very long-lived isomers in EURICA via conversion electron triggered $\gamma$ rays within the $5 \mathrm{~ms}$ to $80 \mathrm{~s}$ range, with the $4 \mathrm{~s}$ isomer in ${ }^{174} \operatorname{Er}$ [21] being observed in this very experiment [22], these very long lived states were searched for but none was observed. While the reduced hindrance of this transition is far below the extrapolated value from ${ }^{172} \mathrm{Er},{ }^{174} \mathrm{Yb},{ }^{176} \mathrm{Hf}$ and ${ }^{178} \mathrm{~W}$, it is worth noting that it is still the second highest in the $N=104$ chain, second only to ${ }^{174} \mathrm{Yb}$. 
The low- $K$ states are less straightforward to interpret than the high- $K$ ones as they are created through an interplay of several different configurations and have a tendency to be collective. In Reference [14] we suggest a $2^{-}$ground state in ${ }^{170} \mathrm{~Tb}$. For $\beta$ decay into a Fermi surface $h_{11 / 2} 5 / 2^{-}$[532] proton the $h_{9 / 2} 5 / 2^{-}$[512] neutron could be a candidate that contribute to the formation of negative parity states as observed in Reference [14]. Coupling this neutron hole with $g_{9 / 2} 1 / 2^{+}[651]$ could create the $2^{-}$and $3^{-}$states observed. However, such a two-neutron configuration would be energetically unfavored by residual interactions, and the $3^{-}$coupling of the same two neutrons would most likely have lower energy.

Another option is that a $h_{11 / 2} \pi 7 / 2^{-}[523] \otimes \pi d_{5 / 2} 3 / 2^{+}[411]$ configuration is populated as a pure two-proton configuration, where the $2^{-}$coupling is energetically favoured, if the ${ }^{170} \mathrm{~Tb}$ ground state is the $d_{5 / 2} \pi 3 / 2^{+}[411] \otimes h_{9 / 2} v 5 / 2^{-}$[512] orbital is the ground state in ${ }^{170} \mathrm{~Tb}$. This would be consistent with the assignment of the $2^{-}$band-head at $1148 \mathrm{keV}$ in ${ }^{162} \mathrm{Dy}[23]$ where this configuration was suggested contribute significantly to the $2^{-}$band. Another interesting notion from ${ }^{162} \mathrm{Dy}$ is that this two-quasi-particle state is expected to be the dominant component of the $2^{-}$octupole vibration. Possible orbitals involved in the formation of $6^{+}$and $2^{-}$states are shown in Figure 4.
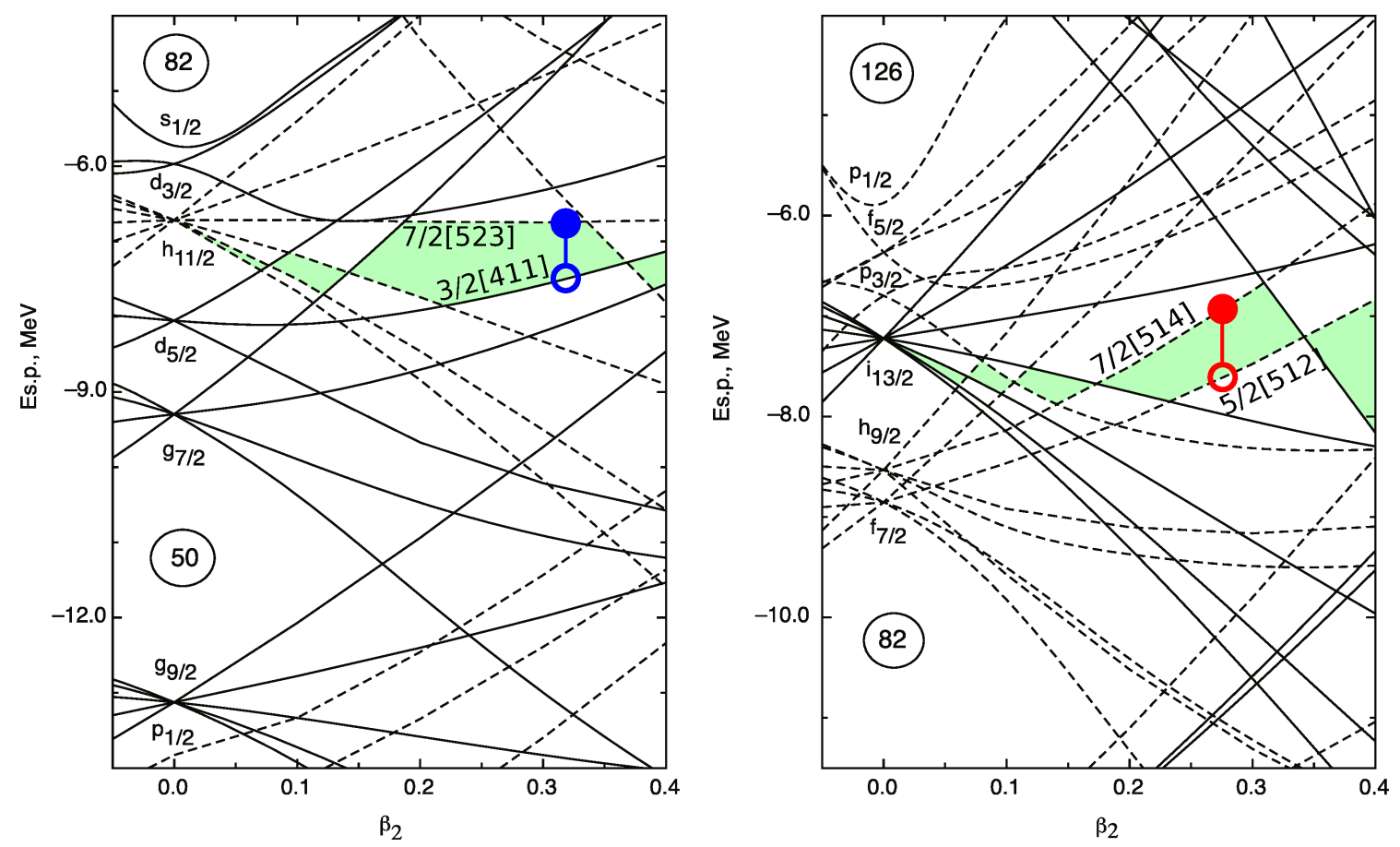

Figure 4: Relevant Nilsson orbitals for protons (left) and neutrons (right) as a function of $\beta_{2}$ deformation. Solid lines represent positive parity and dashed lines negative parity orbitals. The green area represent the Fermi surface of ${ }^{170} \mathrm{Dy}$. Also included are the particle-hole (solid-open circles) states discussed in these proceedings.

In Figure 5 we show potential energy surface calculations in $\left(\beta_{2}, \beta_{4}, \beta_{6}\right)$ deformation space performed for Reference [14]. These calculations give an excitation energy of $1.43 \mathrm{MeV}$ for the $7 / 2^{-}[523] \otimes 3 / 2^{+}[411] 2$-proton configuration in ${ }^{170} \mathrm{Dy}$, while for the calculations in $\left(\beta_{2}, \beta_{3}, \beta_{4}, \beta_{5}\right)$ deformation space, the excitation energy is $1.532 \mathrm{MeV}$. The excitation energy for the $5 / 2^{-}[512] \otimes$ $1 / 2^{+}[651]$ configuration in the $\left(\beta_{2}, \beta_{3}, \beta_{4}, \beta_{5}\right)$ deformation space is $2.132 \mathrm{MeV}$, using an adjusted 
pairing strength, see discussion in Reference [14]. While this discussion consider these configuration in their pure form, it is possible to have a two-proton configuration mixing with a two-neutron configuration of the same spin and parity. This could build up some collectivity, where the lowest state would then be a mixture of both configurations and lower in energy than either would be if unmixed. While the above discussion is also valid for the second $2^{-}$state observed, we note that, using the interacting boson model with an additional $f$ boson, the lowest lying octupole deformed state has been predicted to be a $2^{-}$state at $1.81 \mathrm{MeV}$ [24]. While it is not possible to definitely assign the nature of the second $2^{-}$state as a octupole deformed state or a two-quasi-particle state, of a mix of these, from the current data, both the first and the second $2^{-}$states could be tentative candidates for octupole deformation. This is not in disagreement with the potential surface calculations of the $5 / 2^{-}[512] \otimes 1 / 2^{+}[651]$ state that show a slight increase in $\beta_{3}$ softness.
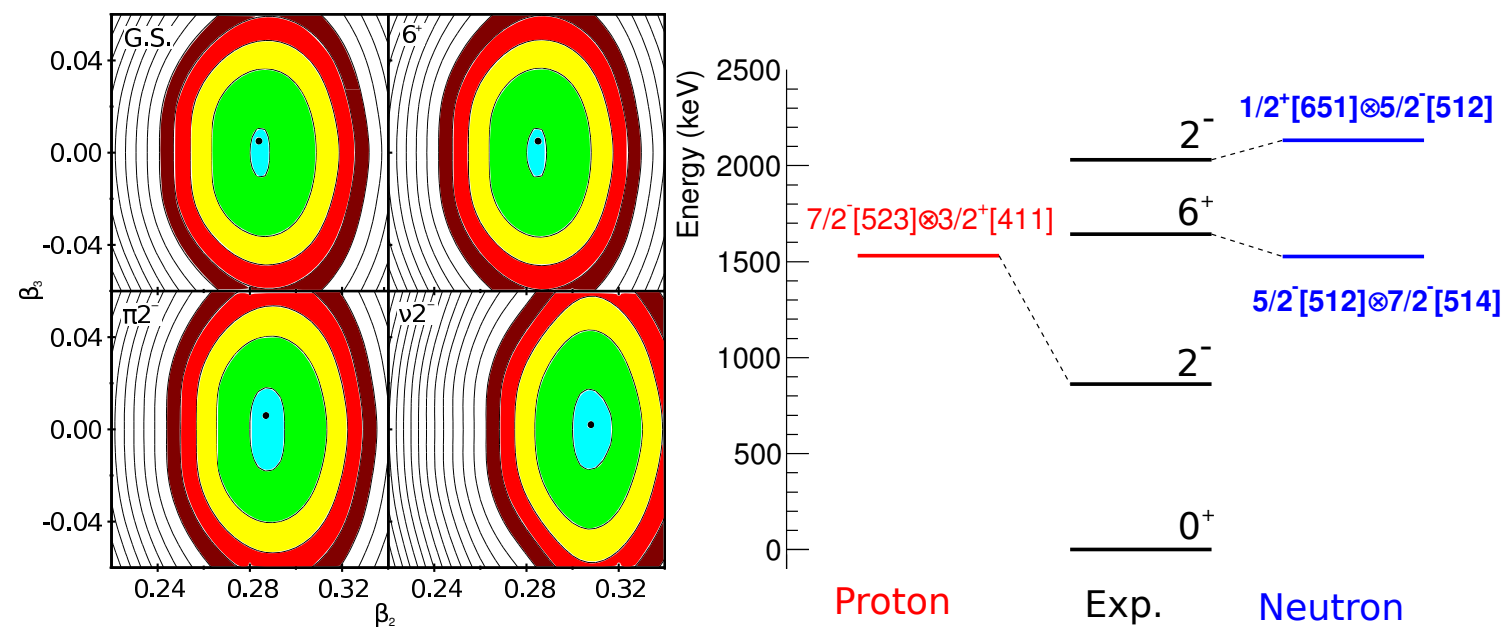

Figure 5: Projections in the $\left(\beta_{2}, \beta_{3}\right)$ plane of the potential energy surfaces (left), minimized in the $\left(\beta_{2}, \beta_{3}, \beta_{4}, \beta_{5}\right)$ space, for the ground state, $v 5 / 2^{-}[512] \otimes v 7 / 2^{-}[514]$ state, $\pi 7 / 2^{-}[523] \otimes \pi 3 / 2^{+}[411]$ state, and $v 5 / 2^{-}[512] \otimes v 1 / 2^{+}[651]$ state. The contours are at $100 \mathrm{keV}$ distance from each other, and the lowest $500 \mathrm{keV}$ contours have been color coded. The energies of the excited states are shown in the right panel with a possible assignment to the experimentally observed states.

\section{Summary}

We have performed a $\gamma$-decay experiment at RIKEN to study ${ }^{170}$ Dy. The experiment was performed using the EURICA setup, which couples the worlds highest intensity in-flight fission facility with a high-efficiency HPGe array. The results from this experiment provide a wealth of information on this nucleus, including the evolution of quadrupole collectivity, rigidity and higher order deformations, as well as the long sought for isomeric $K=6^{+}$state. These results provide us with a rich level scheme for discussing both single-particle and collective structures at mid-shell.

\section{References}

[1] R. F. Casten, P. von Brentano, A. M. I. Haque, Evidence for an underlying SU(3) structure near neutron number N=104, Phys. Rev. C 31 (1985) 1991. 
[2] D. J. Dean, et al, Shell model Monte Carlo calculations for ${ }^{170}$ Dy, Phys. Lett. B317 (1993) 275.

[3] A.K. Rath, et al., Self-consistent description of dysprosium isotopes in the doubly midshell region, Phys. Rev. C 68 (2003) 044315.

[4] C. E. Vargas, V. Velázquez, S. Lerma, Microscopic study of neutron-rich dysprosium isotopes, Eur. Phys. J. A49 (2013) 4.

[5] Zs. Podolyák, et al. Isomer spectroscopy of neutron rich ${ }^{190} W_{116}$, Phys. Lett. B491 (2000) 225.

[6] N. Alkhomashi, et al. $\beta^{-}$-delayed spectroscopy of neutron-rich tantalum nuclei: Shape evolution in neutron-rich tungsten isotopes, Phys. Rev. C 80 (2009) 064308.

[7] P.J.R. Mason, et al. Half-life of the yrast $2^{+}$state in ${ }^{188}$ W: Evolution of deformation and collectivity in neutron-rich tungsten isotopes, Phys. Rev. C 88 (2013) 044301.

[8] S. K. Ghorui, B. B. Sahu, C. R. Praharaj, S. K. Patra, Examining the stability of Sm nuclei around $N=100$, Phys. Rev. C 85 (2012) 064327.

[9] Z. Patel, et al. Isomer Decay Spectroscopy of ${ }^{164} \mathrm{Sm}$ and ${ }^{166} \mathrm{Gd}$ : Midshell Collectivity Around $N=100$, Phys. Rev. Lett. 113 (2014) 262502.

[10] E. Ideguchi, et al. $\mu$ s isomers of ${ }^{158,160}$ Nd, Phys. Rev. C 94 (2016) 064322.

[11] P. Möller, J. R. Nix, W. D. Myers, W. J. Swiatecki, Nuclear Ground-State Masses and Deformations, At. Data Nucl. Data Tab. 59 (1995) 185.

[12] P. H. Regan, et al. Structure of the doubly midshell nucleus ${ }_{66}^{170}$ Dy $y_{104}$, Phys. Rev. C 65 (2002) 037302.

[13] Z. Patel, et al. Decay spectroscopy of ${ }^{160} \mathrm{Sm}$ : The lightest four-quasiparticle K isomer, Phys. Lett. B753 (2016) 182.

[14] P.-A. Söderström, et al. K-mixing in the doubly mid-shell nuclide ${ }^{170}$ Dy and the role of vibrational degeneracy, Phys. Lett. B762 (2016) 404.

[15] T. Kubo, et al. BigRIPS separator and ZeroDegree spectrometer at RIKEN RI Beam Factory, Prog. Theor. Exp. Phys. 2012 (2012) 03C003.

[16] S. Nishimura, Beta-gamma spectroscopy at RIBF, Prog. Theor. Exp. Phys. 2012 (2012) 03 C006.

[17] S. Nishimura, et al. WAS3ABi: The beta-counting system for the EURICA project, RIKEN Accel. Prog. Rep. 46 (2013) 182.

[18] P.-A. Söderström, et al. Installation and Commissioning of EURICA - Euroball-RIKEN Cluster Array, Nucl. Instrum. Meth. B317 (2013) 649.

[19] P.-A. Söderström, et al. Spectroscopy of neutron-rich ${ }^{168,170}$ Dy: Yrast band evolution close to the $N_{p} N_{n}$ valence maximum, Phys. Rev. C 81 (2010) 034310.

[20] P.-A. Söderström, et al. Heavy rotation - evolution of quadrupole collectivity centred at the neutron-rich doubly mid-shell nucleus ${ }^{170}$ Dy, AIP Conf. Proc. 1681 (2015) 030010.

[21] G. D. Dracoulis, et al. Two-quasiparticle K-isomers and pairing strengths in the neutron-rich isotopes ${ }^{174}$ Er and ${ }^{172}$ Er, Phys. Lett. B635 (2006) 200.

[22] P.-A. Söderström, et al. Decay spectroscopy of neutron-rich rare-earth isotopes, RIKEN Accel. Prog. Rep. 48 (2015) 35.

[23] V. G. Solovev, A. V. Sushkov, N. Yu. Shirikova, Low-Lying Nonrotational States in Well Deformed Even-Even Nuclei in the Rare-Earth Region, Fiz. Elem. Chastits At. Yadra 27 (1996) 1643.

[24] Zamfir, N. V., Cottle, P. D., Systematic behavior of octupole states in deformed rare earth nuclei and the interacting boson approximation, Phys. Rev. C 54 (1996) 176. 